\title{
The Role of Robotics in Mitigating Unknown-Unknown Risks: Case of COVID-19
}

\author{
Mounir El Khatib' ${ }^{(0)}$, Hamda Al Falasi², Maryam Al Moosawi², Sara Bin Shafi ${ }^{2}$ \\ ${ }^{1}$ Hamdan Bin Mohammed Smart University, Dubai, United Arab Emirates \\ ${ }^{2}$ Graduate Project Management, Innovation Management, Hamdan Bin Mohammed Smart University, Dubai, United Arab \\ Emirates \\ Email: drmunir.k@gmail.com, Maryam.almoosawi96@gmail.com, Sarabinshafi@gmail.com, Hamda.alaslai@gmail.com
}

How to cite this paper: El Khatib, M., Al Falasi, H., Al Moosawi, M., \& Shafi, S. B. (2021). The Role of Robotics in Mitigating Unknown-Unknown Risks: Case of COVID19. Journal of Service Science and Management, 14, 1-11.

https://doi.org/10.4236/jssm.2021.141001

Received: December 3, 2020

Accepted: January 18, 2021

Published: January 21, 2021

Copyright () 2021 by author(s) and Scientific Research Publishing Inc. This work is licensed under the Creative Commons Attribution International License (CC BY 4.0).

http://creativecommons.org/licenses/by/4.0/

\section{(c) (i) Open Access}

\begin{abstract}
Unknown-unknown risks are unknown risks with unknown consequences. This type of risk is the most difficult to deal with. From this perspective, innovative solutions and technologies are needed like robotics. Many studies dealt with how robotics raise the efficiency and safety levels and provide enhanced levels of service, collect and analyze data for security and safety efforts, and assist in dangerous settings or disaster. This research is based on the core target of identifying the role of robotics in mitigating the impact caused by unknown-unknown risks. The case related to COVID-19 has specifically been considered in this research. This research investigated the application of robotics technology in four organizations. A mixed-method approach involving primary and secondary data collection through interview and secondary literature exploration was used. Literature review suggested that robotics is widely being used in hospitals to offer healthcare-related services. While further research is explored for supporting the responses obtained by selecting two risk managers employed in the selected organizations, based on primary data. The findings of this study state that the managers and the risk management professionals are not trained enough to use robotics to deal with risky scenarios such as COVID-19. Additionally, unknown risks can be managed using AI tools and human intellect-based augmented reality systems. Risk professionals are required to be trained by conducting AI-based workshops for the implementation of desirable and less risk generating technological aids.
\end{abstract}

\section{Keywords}

Robotics, Risks, Unknown Risks, Risk Mitigation, COVID-19

\section{Introduction}

There has been a noticeable increase in the use of automation and robots in the 
healthcare system and other organizations that deal with commodities and tasks, which can be overwhelming for the associated people. The rate of exposure to unknown-unknown risks increases because of the increased innovation levels as well as insufficient knowledge. Moreover, the frequency of unknown-unknown risks is missing due to different reasons such as psychological as well as organizational biases in managing uncertain events (Raydugin, 2018). The occurrence of unknown-unknown risks has been recognized in the healthcare segments despite the constantly evolving technologies. Robots perform realistic risk examination by analyzing the patients' life cycle at the hospital and then applying in-depth reinforcement knowledge for deriving signs of unknown risks (Namba \& Yamada, 2018). However, existing studies have not investigated the effectiveness and implication of robotics, mainly in identifying unknown-unknown risks, specifically in the case of COVID-19, the new ongoing pandemic that is posing a severe threat to the world. Therefore, this study has emphasized examining robotics' implication to reduce the unknown-unknown risks' impact with reference to COVID-19.

\subsection{Research Hypothesis}

Kumar et al. (2020) claimed that modern technology has provided effective measures to fight against COVID-19. Modern technologies' potential has been utilized to tackle both clinical and cultural difficulties generated by COVID-19 (Kumar et al., 2020). In support and as an explanation for this, Chamola et al. (2020) explored the crucial role of Internet of Things (IoT), blockchain, Artificial Intelligence (AI), Unmanned Aerial Vehicles (UAVs), and 5G for mitigating the adverse impacts of COVID-19 outbreak. COVID-19 has affected all the industries, including automobile, aviation, tourism, oil, construction, food, healthcare, and telecommunication. Focusing on healthcare, the proliferation of modern technologies has assisted in treating patients, thereby alleviating stress of healthcare workers, mitigating risk of transmission by undertaking a variety of tasks such as supplying materials, monitoring physical health, building blockchain-enabled apps, and mapping out the occurrence of disease using Geographic Information System (GIS) (Chamola et al., 2020). Centering on this, this research work seeks to test the following hypothesis:

"How technology (Mainly robotics) can mitigate unknown-unknown risks such as that posed by COVID 19?"

\subsection{Aim and Objectives}

\subsubsection{Aim}

To analyze and critically evaluate the effectiveness and contribution of robotics in minimizing the risk posed by unknown-unknown risks by taking COVID-19 as a case.

\subsubsection{Objectives}

- To determine the concept behind unknown-unknown risks and the need for 
mitigating these risks;

- To critically assess the role of robotics in reducing unknown-unknown risks' impact with reference to the case of COVID-19;

- To perform a detailed SWOT analysis of organizations and identify the features of robotics in terms of strengths and opportunities for the organizations to mitigate the risk of threats;

- To highlight the issues in the implementation of robotics and identify ways to overcome such issues to effectively manage unknown-unknown risks.

\subsubsection{Rationale}

It is vital for businesses to overcome unknown-unknown risks. These risks are concerned with facts that not everyone in the organization is aware of; however, the impact on the business and community might be severe (Aven \& Thekdi, 2019; Torres \& Rees, 2017). In context of the healthcare sector, the novelty of COVID-19 pandemic gave rise to the need for hospitals to identify how to combat this virus (Wyman, 2020). The current study therefore focused on determining the way in which advanced technology, mainly robotics, can be beneficial in alleviating the severity of the impacts of COVID-19-related unknown-unknown risks for the welfare of the global community. This study can guide healthcare authorities, risk managers, and healthcare service providers regarding robotics' implication for improving healthcare outcome. Other businesses such as banks and retailers would also gain significant knowledge regarding usage and advantages of robotics in mitigating unknown-unknown risks and improve business efficacy.

\subsection{Organization of Research}

In order to collect the required set of data and information, a systematic structure has been designed for the research work. Table 1 discusses comprehensively the organization of research.

Table 1. Research organization.

\begin{tabular}{|c|c|c|}
\hline No. & Title & Description \\
\hline 1 & Introduction & $\begin{array}{l}\text { This section provides a brief introduction of the research problem } \\
\text { and states the research hypothesis, research aim, objectives, and } \\
\text { research rationale. }\end{array}$ \\
\hline 2 & Literature Review & $\begin{array}{l}\text { This section provides an in-depth review of existing literary sources } \\
\text { and other credible sources to generate a robust theoretical base for } \\
\text { the research topic. }\end{array}$ \\
\hline 3 & $\begin{array}{l}\text { Methodology and } \\
\text { Expected Findings/ } \\
\text { Analysis }\end{array}$ & $\begin{array}{l}\text { This chapter discusses the methods used for addressing the research } \\
\text { aims and objectives, followed by an analysis of the gathered data to } \\
\text { draw meaningful conclusions. }\end{array}$ \\
\hline 4 & $\begin{array}{l}\text { Recommendations and } \\
\text { Conclusion }\end{array}$ & $\begin{array}{l}\text { Based on the findings drawn, a range of recommendations have been } \\
\text { made in this section to foster the implementation of robotics. } \\
\text { Following this, the conclusion drawn from this work has been } \\
\text { presented. }\end{array}$ \\
\hline
\end{tabular}




\section{Literature Review}

\subsection{Concept behind Unknown Risk in Organizations}

As mentioned by Kucharski (2020), an important responsibility of a risk analyst is to predict the unexpected issue and prepare the organization to deal with it effectively and efficiently. In case of unforeseen events, the management turns to risk managers to find a solution. For a plan to be designed in advance, sufficient preparation and time are required. While no risk manager can anticipate a virus outbreak, previous outbreaks such as SARS can be used as an example for designing a contingency plan that might work out in an environment of uncertainty.

\subsection{Importance of Robotics in Reducing Unknown Risk}

According to Kilpatrick and Barter (2020), robots can act as a barrier between a doctor and a patient, thereby reducing the human interaction and consequent risk of infection transmission. Robots are also being used to offer counselling and assistance to quarantined patients. At the Hongshan Sports Centre in $\mathrm{Wu}$ han, China, a field hospital with 14 robots has been opened. The robots supplied by Cloud Minds from Peking are intended to purify and disinfect, distribute medication, and measure the temperature levels of patients (Kimmig et al., 2020). Figure 1 below shows the reported use of robots worldwide for COVID-19 as of April 2020. Figure 1 also shows the number of instances based on modality. At this time, only ground and aerial modalities have been reported. Of the 203 instances, 119 are ground and 84 are aerial.

74

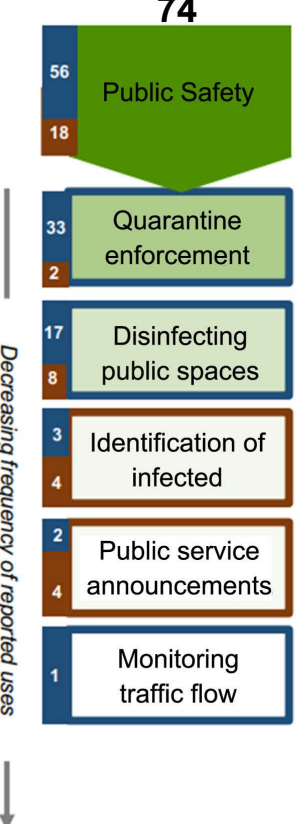

46

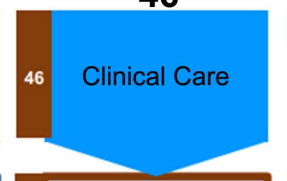

Healthcare telepresence

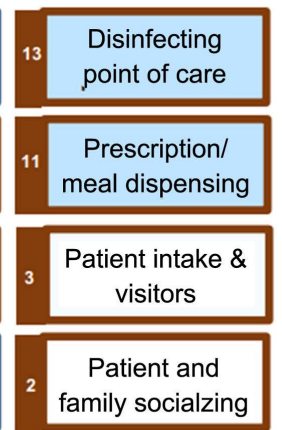

Inventory
22

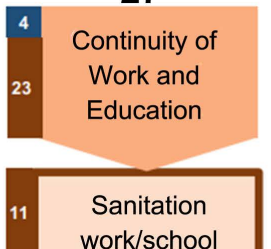
work/school
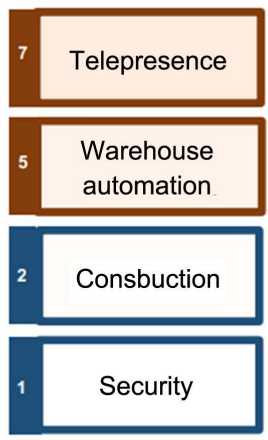

Agriculture
21
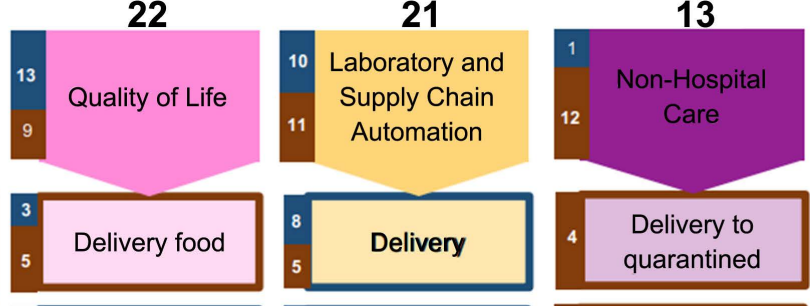

Delivery to quarantined
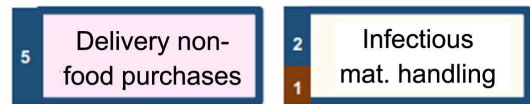

Quarantine socializing
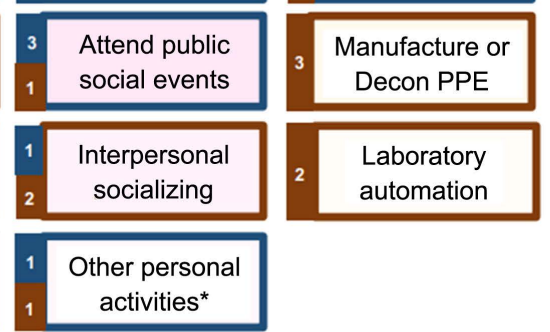

Off-Site Testing

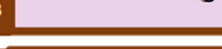

Testing, care in nursing homes

Figure 1. 203 instances of robot use for COVID-19 applications organized into six categories and by modality, with brown representing ground robots and blue, aerial robots. 


\subsection{Implementation of Robotics Technology and Identification of Its Unknown Risk}

Robots telehealth service allows doctors to interact with patients virtually, thereby reducing resource consumption and encouraging infectious patients to stay confined. Robots can interact not only with individuals who contract the coronavirus infection but also gain valuable medical knowledge to help doctors manage patients. Because of COVID-19, it has become necessary to minimize the interaction between human beings (Prettner \& Bloom, 2020).

- Technical firms have employed robots in several places, such as in medical settings, because they are resistant to infection.

- Chinese hospitals have employed robots to disinfect rooms; thus, if fully deployed, the robots can work in every Chinese province.

- Robots use UV light to destroy viruses and bacteria without exposing humans to infection. Such bots are operated by health workers remotely, that is, from a secure distance away (Iansiti \& Lakhani, 2020).

\section{Methodology}

\subsection{Data Collection}

This study explored multi-dimensional data segments to enable reviewing of the required specific data about the use of robots for dealing with unknown-unknowns risks. Thus, a mixed-method approach was used for acquiring data. The interview method offered broad and descriptive data. Secondary data sources were reviewed to identify supportive arguments presented by other researchers; this further helped in validating the primary data (Brannen, 2017).

\subsection{Sampling}

For the interview, two risk managers from Dubai Silicon Oasis Authority and HSBC bank were chosen using convenient/selective sampling. The reason for interviewing risk managers for this research work was to highlight the current unknown-unknown risks that have emerged during this COVID-19 crisis and devise efficient mitigation strategies. In addition, a SWOT analysis was performed to study the efficacy of robots in tackling unknown-unknown risks faced by their organizations. For secondary data collection, two hospitals, Third People's Hospital in Shenzhen and Wuhan Wuchang Hospital, were selected as samples to further add knowledge about the robotics' ability to mitigate unknown-unknown risks associated with COVID-19.

\subsection{Analysis}

For analyzing the mixed data sets retrieved using the two diverse methods as well as the interview, a narrative analysis was performed. The data gathered using the primary and secondary method were classified based on their related themes and were analyzed by associating them with the literature (Guest et al., 2011). 


\subsection{Expected Findings/Analysis}

\subsubsection{Theme 1: Determination of the Concept Associated with Unknown-Unknown Risks and the Need to Mitigate Such Risks}

From the primary findings gathered through interview, it is understood that risks falling in the unknown-unknown category are being observed because of the usage of technological aids in organizational practices. In Dubai Silicon Oasis, it was observed that the usage of electronic form of communication network by the organization has led to unknown risks of the cybercrime. Furthermore, the ongoing pandemic has generated unknown risks to the well-being and safety levels of the workers. Apart from this, as per the secondary findings, it was observed that for mitigating the unknown risks, Dubai Silicon Oasis uses risk management plan, including major risk resolving activities that are focused on prohibiting the occurrence of the risk, mitigation of the risk, transferring as well as accepting the risk.

\subsubsection{Theme 2: Critical Examination of Effectiveness of Role and Implication of Robotics}

It can be observed that COVID-19 has incrementally increased unknown risks, mainly for healthcare organizations. While evaluating the secondary data on the case of Wuhan Wuchang Hospital, it was noted that in this hospital, the unknown risks concerned with COVID-19 increased because of the lack of knowledge and awareness regarding this disease's nature. As the death rates and number of positive cases constantly increased, the hospital failed to handle the situation due to lack of required facilities. Moreover, Wuhan-based hospitals lack sufficient resources, which makes the current situation of COVID-19 further challenging (Zhang et al., 2020). In this regard, secondary findings also show that for reducing unknown-unknown risks' effects, robots would need to identify common signs and symptoms of COVID-19 in patients in the initial phase so as to ensure effective treatment. The usefulness of robots is also evident from the fact that its intelligence is being used to assess the temperature and heart rate of patients and keep the records updated for the staff. Another practical implication of robotics is that robotics-based devices support hospital staff to keep infectious diseases such as COVID-19 from spreading to the community.

Further, taking the case of Third People's Hospital of Shenzhen as another example, robots can help in diagnosing the patients and alerting the healthcare providers in case of any emergencies (Clifford, 2020).

In case of financial industries, one can take the example of HSBC, which has implemented remote working to maintain business processes smoothly while also managing employees' safety (HSBC, 2020). HSBC mentioned that it will make an investment of up to $\$ 17$ billion in technology by 2020 . This is to enhance the experiences of customers and to ensure a modernized transformation and interaction. In addition, the implementation of robo-advisers will help ensure customer satisfaction along with keeping the customers, as well the business safe. Furthermore, robo-advisers can perform tasks faster than regular relation- 
ship managers, which would ensure customer satisfaction. Investing in technology aids an organization to become more adaptive to changes (Bloomberg, 2018).

The use of advanced technology for fulfilling customers' need is clear from the case of Dubai Silicon Oasis. Various practical benefits of using robots have been observed in terms of mitigating unknown-unknown risks of COVID-19 in different industry-based organizations.

\subsubsection{Theme 3: Highlighting the Issues for Implementing the Robotics}

As per the primary findings, robots are being used in the healthcare segments of China to manage the unknown-unknowns risks in relation to the COVID-19 outspread. Robotic devices are used to maintain distancing and treating infected patients in a less infection-generating environment. However, implementation of robotic aids faces issues such as lack of collaboration among the healthcare providers. Also, healthcare providers are not skilled enough to manage the robotic equipment and AI tools designed to deal with unfamiliar scenarios created because of the Coronavirus pandemic. Further, the secondary data sources have also shown that virtual reality and AI can be used as techniques for dealing with the problems of unknown-unknowns risks. In AI-based equipment designed to monitor the symptoms of COVID-19, the infected person is scanned through virtual reality platforms to enhance the effectiveness of reducing the unknown risk of spreading the virus among healthcare providers.

Regarding the strength of Wuhan Wuchang Hospital, it was observed that the assistance provided by robotics helped in determining the signs of COVID-19 in patients, sharing the burden of the staff, and providing entertainment at the facilities for quarantined patients (Hornyak, 2020). On the other hand, at Third People's Hospital of Shenzhen, robots helped in enhancing the daily productivity of hospitals and enabling patients to make decisions about their healthcare needs.

Modern technology adoption has resulted in satisfaction of Dubai Silicon Oasis' customers. It was also observed that other organizations such as HSBC have the opportunity to use robots to detect important signs and symptoms of COVID-19 in suspected employees to prevent infection transmission and refer the infected ones to hospitals for proper treatment (Hornyak, 2020).

From the findings it is clear that in order to develop measures to reduce the unknown risks of COVID-19, businesses and healthcare organizations should make a substantial investment in the newer technologies such as augmented reality and AI. These technologies enhance the prediction of uncertain scenarios that are more likely to occur and further offer the opportunity for decision making by the managerial unit to identify and resolve the possible, unknown risk-generating occurrences (Clifford, 2020).

The results of the study show that there exist several risks associated with the fewer numbers of trained workers, along with weak collaboration between the risk managers and the risk-mitigating technological devices. Further, the absence 
Table 2. SWOT Analysis Based on Interviews of Primary and Secondary data.

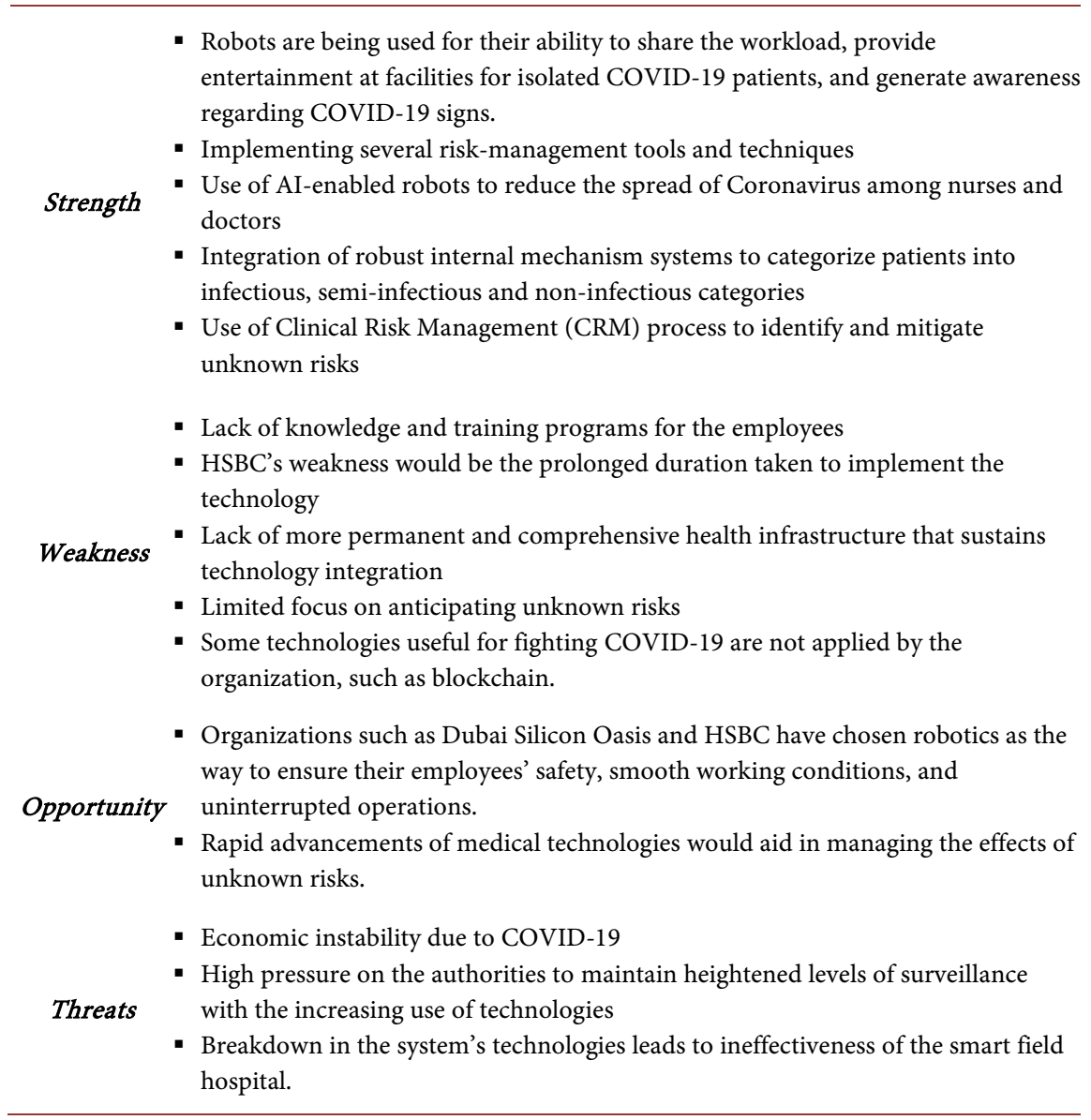

of technological implementation reduces the efficacy of robots in dealing with adverse situations (Media, 2020). Table 2 demonstrates the SWOT Analysis Based on Interviews of Primary and Secondary data.

\section{Recommendations and Conclusion}

\subsection{Recommendations}

- Robotic technology can be effectively implemented in organizations; leaders must come up with ways to use using robots and significantly influence their employees into accepting it so as to bring about this technology-oriented change for preventing rising cases of COVID- 19 .

- It is recommended that workshops and training programs based on technologies such as robotics, AI, and augmented reality are conducted to educate the employees on how to use these modern aids for risk management within an organization. It will further help in increasing the efficacy rate of this technological support for managing any crisis in the future.

- Organizations must have contingency plans that implement modern technologies such as robotics, augmented reality, and AI in order to help with the prediction of potential risks along with the unknown impacts they might 
have on businesses and health and wellbeing.

- It is recommended that organizations create a new culture among their employees to educate them about the importance of robotics in mitigating unknown risks through training programs and awareness.

\subsection{Conclusion}

In conclusion, robotics has a wide range of benefits and can make a significant contribution to diminish the effects of unknown-unknown risks and help in mitigating such risks, especially with regard to COVID-19. The secondary data clearly reflects that robots can help in identifying COVID-19 symptoms and raising attention among healthcare providers to assist the patients, allowing smooth day-to-day functioning of the hospitals and managing the burden of healthcare providers by effectively managing COVID-19 cases in an automated manner.

Based on the SWOT analysis, the strengths of implementing robotics in organizational settings identified were their ability to share the workload, provide entertainment at facilities for isolated COVID-19 patients, and generate awareness regarding COVID-19 signs and symptoms. Organizations such as Dubai Silicon Oasis and HSBC have chosen robots, as it guarantees their employees' safety, smooth working conditions, and uninterrupted operations. From the outcomes of this research, effective ways to manage unknown risks have been identified, such as the use of technologies, augmented reality as well as AI. Further, this study can be used effectively to improve the skills of the healthcare workers and risk managers in using and implementing robotic technology for treating the patients during the COVID-19 pandemic and thus reducing the prevalence of unknown risk-based conditions in the future.

In addition to the findings of the study, a wider scope for future research has been made available on the topic with regard to the disastrous impact of COVID-19 on economic, social as well as health dimensions. For future research, studies must be conducted at a massive level involving a substantial sample of population and wider data on the topic. Along with this, a mixed research method can be used that will help in collecting both qualitative and quantitative data on the research topic, using survey and interview method both. Additionally, a scope of narrowing down the research topic is available on the topic, as currently several organizations have been included in the study. In this context, a specific area can be chosen, such as banking and financial industry.

\section{Conflicts of Interest}

The authors declare no conflicts of interest regarding the publication of this paper.

\section{References}

Aven, T., \& Thekdi, S. (2019). Enterprise Risk Management: Advances on Its Foundation 
and Practice. Abingdon-on-Thames: Routledge.

Bloomberg (2018). New CEO’s \$17 Billion Plan for HSBC: Expand Key ASIAN Markets, Improve Tech.

https://www.businesstimes.com.sg/banking-finance/hsbcs-new-chief-outlines-plan-tospend-us17-billion-by-2020\#: :text=HSBC\%20Holdings\%20Plc\%20Chief\%20Executiv e,the $\% 20$ helm\%20four\%20months\%20ago

Brannen, J. (2017). Mixing Methods: Qualitative and Quantitative Research. Abingdon-on-Thames: Routledge.

Chamola, V., Hassija, V., Gupta, V., \& Guizani, M. (2020). A Comprehensive Review of the COVID-19 Pandemic and the Role of IoT, Drones, AI, Blockchain, and 5G in Managing its Impact. IEEE Access, 8, 90225-90265.

https://doi.org/10.1109/ACCESS.2020.2992341

Clifford, C. (2020). Look Inside the Hospital in China Where Coronavirus Patients Were Treated by Robots.

https://www.cnbc.com/2020/03/23/video-hospital-in-china-where-covid-19-patients-tr eated-by-robots.html

Guest, G., MacQueen, K. M., \& Namey, E. E. (2011). Applied Thematic Analysis. Thousand Oaks, CA: Sage Publications. https://dx.doi.org/10.4135/9781483384436

Hornyak, T. (2020). What America Can Learn from China's Use of Robots and Telemedicine to Combat the Coronavirus.

https://www.cnbc.com/2020/03/18/how-china-is-using-robots-and-telemedicine-to-co mbat-the-coronavirus.html

Hongkong and Shanghai Banking Corporation Limited (2020). Novel Coronavirus: Customer Support and Our Action Plan.

https://www.business.hsbc.co.in/en-gb/in/article/novel-coronavirus

Iansiti, M., \& Lakhani, K. R. (2020). Competing in the Age of AI: Strategy and Leadership When Algorithms and Networks Run the World. Brighton, MA: Harvard Business Press.

Kilpatrick, J., \& Barter, L. (2020). COVID-19: Managing Supply Chain Risk and Disruption.

https://www2.deloitte.com/content/dam/Deloitte/ca/Documents/finance/Supply-Chain POV_EN_FINAL-AODA.pdf

Kimmig, R., Verheijen, R. H. M., Rudnicki, M., \& SERGS Council (2020). Robot Assisted Surgery during the COVID-19 Pandemic, Especially for Gynecological Cancer: A Statement of the Society of European Robotic Gynaecological Surgery (SERGS). Journal of Gynecologic Oncology, 31, e59. https://doi.org/10.3802/jgo.2020.31.e59

Kucharski, A. (2020). The Rules of Contagion: Why Things Spread-And Why They Stop. New York, NY: Basic Books.

Kumar, A., Gupta, P. K., \& Srivastava, A. (2020). A Review of Modern Technologies for Tackling COVID-19 Pandemic. Diabetes \& Metabolic Syndrome: Clinical Research \& Reviews, 14, 569-573.

Media, H. (2020). Roundup: Tech's Role in Tracking, Testing, Treating COVID-19. https://www.mobihealthnews.com/news/roundup-techs-role-tracking-testing-treatingcovid-19

Namba, T., \& Yamada, Y. (2018). Risks of Deep Reinforcement Learning Applied to Fall Prevention Assist by Autonomous Mobile Robots in the Hospital. Big Data and Cognitive Computing, 2, 13-27. https://doi.org/10.3390/bdcc2020013

Prettner, K., \& Bloom, D. (2020). Automation and Its Macroeconomic Consequences. 
London: Academic Press.

Raydugin, Y. G. (2018). Holistic View on Unknown Unknowns in Project Risk Management. In M. Tavana (ed.), Developing Business Strategies and Identifying Risk Factors in Modern Organizations (pp. 82-93). Hershey, PA: IGI Global. http://doi:10.4018/978-1-4666-4860-9.ch006

Torres, P., \& Rees, M. (2017). Morality, Foresight, and Human Flourishing: An Introduction to Existential Risks. London: Pitchstone Publishing.

Wyman, O. (2020). More Risks, More Unknowns.

https://www.oliverwyman.com/our-expertise/insights/2020/apr/risk-journal-vol-9.html

Zhang, J., Wang, M., Zhao, M., Guo, S., Xu, Y., Ye, J. et al. (2020). The Clinical Characteristics and Prognosis Factors of Mild-Moderate Patients with COVID-19 in a Mobile Cabin Hospital: A Retrospective, Single-Center Study. Frontiers in Public Health, 8, 264. https://doi.org/10.3389/fpubh.2020.00264 